\title{
Clinical Guideline Highlights for the Hospitalist: Diagnosis and Management of Measles
}

\author{
Marie E Wang, MD, MPH ${ }^{1 *}$, Adam J Ratner, MD, $\mathrm{MPH}^{2}$
}

'Department of Pediatrics, Stanford University School of Medicine, Stanford, California; ${ }^{2}$ Departments of Pediatrics and Microbiology, New York University School of Medicine, New York, New York.

GUIDELINE TITLE: (1) Measles (Rubeola): For Healthcare Professionals and (2) Interim Infection Prevention and Control Recommendations for Measles in Healthcare Settings

RELEASE DATE: (1) February 5, 2018, and (2) July 2019 PRIOR VERSION(s): n/a
DEVELOPER: Centers for Disease Control and Prevention (CDC)

FUNDING SOURCE: CDC

TARGET POPULATION: Children and adults with suspected or confirmed measles

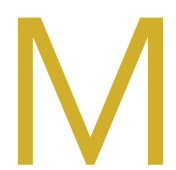

easles is a highly contagious acute respiratory illness that can cause complications in multiple organ systems. Measles was declared eliminated in the United States in 2000; however, outbreaks still occur, especially in unvaccinated populations. The Centers for Disease Control and Prevention (CDC) reported that as of October 3, 2019, 1,250 cases of measles had been confirmed in 31 states in 2019, which represents the greatest number of cases reported in the US since $1992 .^{1}$ Although the disease is often self-limited, infected individuals can also develop complications requiring hospitalization, which occurred in $10 \%$ of confirmed cases this year. ${ }^{1}$ In February 2018, the CDC updated their recommendations about measles diagnosis and treatment on their website, ${ }^{2}$ adding an interim update in July 2019 to include new guidelines about infection control and prevention. ${ }^{3}$ This highlight reviews those recommendations most relevant to hospitalists, who can play a critical role in the diagnosis and management of patients with suspected and/or confirmed measles.

\section{KEY RECOMMENDATIONS FOR THE HOSPITALIST}

Recommendation 1. Healthcare providers should consider measles in patients presenting with febrile rash illness and clinically compatible measles symptoms, especially if the person recently traveled internationally or was exposed to a person with febrile rash illness. Healthcare providers should report suspected measles cases to their local health department within 24 hours.

Measles is an acute febrile illness that begins with a prodrome of fever, followed by one or more of the following three

*Corresponding Author: Marie E. Wang, MD, MPH; E-mail: marie.wang@ stanford.edu; Telephone: 650-736-4423.

Published online first December 18, 2019

Received: September 24, 2019; Revised: October 17, 2019;

Accepted: October 20, 2019

(c) 2020 Society of Hospital Medicine DOI 10.12788/jhm.3346
"C's": cough, coryza (rhinitis), and conjunctivitis. Koplik spots, a pathognomonic buccal enanthem consisting of white lesions on an erythematous base, can appear shortly thereafter. An erythematous, maculopapular rash develops three to four days after the onset of the fever. The rash starts on the face and then spreads over the next few days to the trunk and extremities. Clinical recovery generally occurs within one week of rash onset in uncomplicated measles. Complications can affect almost any organ system. The most common complications are pneumonia, often caused by secondary viral or bacterial pathogens, diarrhea, otitis media, and laryngotracheobronchitis. Rare but serious complications include acute encephalitis and subacute sclerosing panencephalitis. Groups at the highest risk for serious disease include children aged $<5$ years, adults aged $>20$ years, pregnant women, and immunocompromised individuals.

When encountering patients with a febrile rash and compatible symptoms, clinicians should also have a high index of suspicion for measles in patients who are unvaccinated or undervaccinated, since the majority of measles cases have occurred in the unvaccinated population. Providers should contact their local health department and infectious diseases/infection control team as soon as suspected measles cases are identified. Laboratory confirmation is necessary for all suspected cases and should typically consist of measles IgM antibody testing from serum and real-time polymerase chain reaction (RT-PCR) from respiratory and urine specimens.

Recommendation 2. Adhere to airborne precautions for anyone with known or suspected measles.

Measles is highly contagious, and infectious particles can remain in the air for up to two hours after a person with measles leaves a room. From 2001 to 2014, 6\% (78/1,318) of nonimported measles cases in the US were transmitted in healthcare settings. ${ }^{4}$ Key steps in preventing the spread of measles within hospitals include ensuring that all healthcare personnel have evidence of immunity to measles and rapid identification and isolation of suspect cases. Patients with suspected measles should be given a facemask and moved immediately into 
a single-patient airborne infection isolation room. Personnel, even those with presumptive evidence of immunity, should use N95 respirators or the equivalent when caring for patients with suspected or confirmed measles. Patients with measles are contagious from four days before to four days after rash onset; therefore, airborne precautions should be continued for four days following the onset of rash in immunocompetent patients. For immunocompromised patients, airborne precautions should be continued for the duration of the illness based on data suggesting prolonged shedding, particularly in the setting of altered T-cell immunity. ${ }^{4}$

Recommendation 3. People exposed to measles who cannot readily show that they have evidence of immunity against measles should be offered postexposure prophylaxis (PEP) or be excluded from the setting (school, hospital, childcare). To potentially provide protection or modify the clinical course of disease among susceptible persons, either administer a measles, mumps, and rubella (MMR) vaccine within 72 hours of initial measles exposure or immunoglobulin (IG) within six days of exposure.

MMR vaccine is recommended for vaccine-eligible, exposed individuals aged $\geq 6$ months within 72 hours of measles exposure. IG, which contains measles antibody due to widespread immunization in the US, is recommended for individuals at high risk for serious illness, including infants aged $\leq 12$ months, pregnant women without evidence of measles immunity, and severely immunocompromised patients regardless of vaccination status. For infants aged 6-11 months, MMR vaccine can be given in place of IG if done within 72 hours of exposure. PEP for children during the 2013 New York City outbreak reduced the risk of measles by $83.4 \%$ (95\% Cl: 34.4\%-95.8\%) in recipients of MMR vaccine and by $100 \%$ (95\% Cl: $56.2 \%-99.8 \%$ ) in recipients of IG compared with those without prophylaxis. ${ }^{5} \mathrm{~A}$ 2014 Cochrane Review found that IG reduced the risk of measles by $83 \%$ (95\% Cl: 64\%-92\%). ${ }^{6}$

Recommendation 4. Severe measles cases among children, such as those who are hospitalized, should be treated with vitamin A. Vitamin A should be administered immediately on diagnosis and repeated the next day.

In children, vitamin A deficiency, even if clinically inapparent, leads to increased measles severity, and randomized controlled trial data suggest that supplementation reduces measles-related morbidity and mortality. ${ }^{4}$ Even in high-income countries, children with measles have high rates of vitamin A deficiency, which is associated with increased morbidity. ${ }^{7}$ A Cochrane review found that two-dose regimens of vitamin A reduced the overall mortality (RR $0.21 ; 95 \% \mathrm{Cl}$ : 0.07-0.66) in children with measles aged $<2$ years. ${ }^{8}$ World Health Organization guidelines suggest vitamin A therapy for all children with acute measles infection, and the AAP Committee on Infectious Diseases recommends vitamin A for severe (ie, hospitalized) cases. Vitamin A is given orally once daily for two days at the following doses: 50,000 international units (IU) for infants aged $<6$ months, 100,000 IU for infants aged 6-11 months, and 200,000 IU for children aged $\geq 12$ months. A third dose can be given two to four weeks later for children with signs and symptoms of vitamin A deficiency (eg, corneal clouding or conjunctival plaques).

\section{CRITIQUE}

In outbreak settings, hospitalists may find challenges with having a sufficient number of single negative-pressure rooms for patients with suspected or confirmed measles and providing IG prophylaxis given the recent national shortages of intravenous immunoglobulin. Collaboration with the infection control team, pharmacy, and the local public health department is essential to appropriately address these challenges. With regard to treatment recommendations, randomized studies on the impact of vitamin A treatment in children have been primarily conducted in resource-limited settings. ${ }^{8}$ However, these data, in combination with observational data from resource-rich settings, ${ }^{7}$ support its use given the favorable risk-benefit profile. The role of vitamin A therapy in adults with measles infection is considerably less clear, although there are reports of its use in severe cases.

\section{AREAS OF FUTURE STUDY}

Much of our knowledge regarding measles complications and treatment outcomes comes from resource-limited settings or from older data before widespread vaccination. Data suggest that prophylactic antibiotics may prevent complications; however, currently available data are insufficient to support routine use. ${ }^{9}$ Coordination and collaboration between public health, infectious diseases, and hospital medicine would enhance the ability to conduct detailed epidemiologic studies during outbreak situations. Further studies examining treatment and outcomes in hospitalized patients, including the role of prophylactic antibiotics in the prevention of complications, would provide valuable guidance for hospitalists caring for patients with severe measles.

Disclosures: Dr. Wang has nothing to disclose. Dr. Ratner has served as a consultant for Pfizer outside the scope of this work.

\section{References}

1. Centers for Disease Control and Prevention. Measles Cases and Outbreaks. 2019; https://www.cdc.gov/measles/cases-outbreaks.html. Accessed October 14, 2019.

2. Centers for Disease Control and Prevention. Measles (Rubeola): For Healthcare Professionals. 2019; https://www.cdc.gov/measles/hcp/index.html. Accessed October 14, 2019.

3. Centers for Disease Control and Prevention. Interim Infection Prevention and Control Recommendations for Measles in Healthcare Settings. 2019.

4. Fiebelkorn AP, Redd SB, Kuhar DT. Measles in healthcare facilities in the United States during the postelimination era, 2001-2014. Clin Infect Dis. 2015;61(4):615-618. https://doi.org/10.1093/cid/civ387.

5. Arciuolo RJ, Jablonski RR, Zucker JR, Rosen JB. Effectiveness of measles vaccination and immune globulin post-exposure prophylaxis in an outbreak setting-New York City, 2013. Clin Infect Dis. 2017;65(11):1843-1847. https:// doi.org/10.1093/cid/cix639.

6. Young MK, Nimmo GR, Cripps AW, Jones MA. Post-exposure passive immunisation for preventing measles. Cochrane Database Syst Rev. 2014;(4):Cd010056. https://doi.org/10.1002/14651858.CD010056.pub2.

7. Frieden TR, Sowell AL, Henning KJ, Huff DL, Gunn RA. Vitamin A levels and severity of measles. New York City. Am J Dis Child. 1992;146(2):182-186. https://doi.org/10.1001/archpedi.1992.02160140048019

8. Huiming $Y$, Chaomin W, Meng M. Vitamin A for treating measles in children. Cochrane Database Syst Rev. 2005(4):Cd001479. https://doi. org/10.1002/14651858.CD001479.pub3

9. Kabra SK, Lodha R. Antibiotics for preventing complications in children with measles. Cochrane Database Syst Rev. 2013(8):Cd001477. https://doi. org/10.1002/14651858.CD001477.pub3. 